\title{
The Production of Boron Carbide by Carbothermic Reduction
}

\author{
G. Goller, C. Toy*, A. Tekin and C.K. Gupta** \\ Faculty of Chemistry and Metallurgy, Istanbul Technical University, Maslak, Istanbul, Turkey \\ *TUBITAK-Marmara Research Center, Materials Research Department, Gebze, Kocaeli, Turkey \\ **Materials Group, Bhabha Atomic Research Center, Bombay, India.
}

\begin{abstract}
Carbothermic reduction of boric acid has scientific and economic advantages over other methods of boron carbide production. The strongly endothermic reaction which has a favourable free energy change only at high temperatures is best carried out in a specially designed graphite resistance furnace at temperatures above $2000^{\circ} \mathrm{C}$, using boric acid and petroleum coke as starting materials. The composition of the charge has a strong influence on the quality of boron carbide obtained and also on the yield. The use of boron anhydride in place of boric acid decreases the yield. The yield is also affected by the heating conditions prevailing in the furnace. The yield of boron carbide can be substantially improved and thus the specific energy consumption in boron carbide production can be significantly decreased by adding an optimum quantity of salt to the boric acid-petroleum coke mixture. The various factors that influence the quality and yield of boron carbide product and hence the economy of boron carbide production by the carbothermic reduction route have been discussed in this paper.
\end{abstract}

\section{INTRODUCTION}

Boron carbide is a specially hard material in the group of technical ceramics. It has found many applications in different areas due to its extreme hardness (2900-3100 kg mm $\left.\mathrm{mm}^{-2}\right)$, low density $\left(2.54 \mathrm{~g} \mathrm{cc}^{-1}\right)$ and good neutron absorption cross section (600 barns). The hardness of boron carbide has made it a useful material for use as an abrasive in polishing and grinding media applications. Besides, boron carbide is cheap. Sintered boron carbide shapes find use as wear-resistant components in sand blasting and water jet cutting nozzles, hard ceramic bearings and wire drawing dies. The combination of extreme hardness and low density of boron carbide has made it a suitable material for uses such as light-weight boron carbide armour plates in helicopters and fighter planes. On account of its favourable neutron absorption characteristics both in the thermal and epithermal ranges, boron carbide is the material of choice for use in neutron control and shielding applications in the nuclear industry. Besides, the light weight coupled with a large heat of combustion $(51900 \mathrm{~J} / \mathrm{g})$ of boron carbide makes it a useful solid propellant for rockets. Boron carbide also has certain useful properties as a material which can resist high temperature chemical attack.

Boron carbide can be directly synthesized from its elements boron and carbon. The high cost of elemental boron, however, makes this route economically unattractive. Boric acid $\left(\mathrm{H}_{3} \mathrm{BO}_{3}\right)$ and boron anhydride $\left(\mathrm{B}_{2} \mathrm{O}_{3}\right)$ are inexpensive starting materials from which boron carbide may be produced by reduction in the presence of or by using carbon. Both metallothermic and carbothermic processes have been used. The reduction of boron anhydride by magnesium in the presence of carbon yields fine amorphous boron carbide powder $/ 1-3 /$. The secondary milling operation is minimised. By carbothermic reduction, the conversion 
of boron anhydride to boron carbide occurs in the following two stages:

$$
\begin{aligned}
& \mathrm{B}_{2} \mathrm{O}_{3}+3 \mathrm{CO}=2 \mathrm{~B}+3 \mathrm{CO}_{2} \\
& 4 \mathrm{~B}+\mathrm{C}=\mathrm{B}_{4} \mathrm{C}
\end{aligned}
$$

The first stage reduction becomes thermodynamically feasible above $1400^{\circ} \mathrm{C}$. However, a temperature beyond $2000^{\circ} \mathrm{C}$ has been recommended /4/ for an enhanced rate of reduction accompanied by the formation of $\mathrm{B}_{4} \mathrm{C}$. The process is highly endothermic, needing 16800 $\mathrm{kJ} / \mathrm{mol}$ or $9.1 \mathrm{kWh} / \mathrm{kg}$ of $\mathrm{B}_{4} \mathrm{C}$. The reduction may be carried out in an electric arc furnace or in a resistance furnace. In the electric arc furnace pellets of $\mathrm{B}_{2} \mathrm{O}_{3}-\mathrm{C}$ mixtures are placed in the central zone of the electric arc and they become heated to $2500^{\circ} \mathrm{C} / 4 /$. Boron carbide crystallises out from the molten phase. Boron carbide produced this way contains a high amount, 10 to $12 \%$, of free carbon. This is due to the volatilisation of boron at such very high reaction temperatures. Boron carbide with so much free carbon is unsuitable for certain applications, e.g., in the nuclear industry.

Carbon-free boron carbide can be readily prepared from a boric acid-carbon mixture in a furnace utilising a graphite rod as the resistive load. Similar to the SiC production furnace, a central graphite rod is used to supply energy to the system for carbothermic reduction. The charge is in the form of a powder mixture. While the furnace is in operation, the empty space that formed within the charge bed due to evaporation of water vapour can be filled with recycled charge from previous runs. Since the temperatures reached in this furnace are much lower than those in the electric arc furnace, boron loss by volatilisation is significantly less and free carbon content in the product is easily controlled.

In another graphite resistive load furnace, a graphite tube is the heating element and the reduction is carried out in a protective atmosphere to form finegrain boron carbide powder $15 /$. The carbothermic reduction process is generally more amenable to control than the magnesiothermic reduction even though the thermite process requires lower investment and milling operation costs. Powders prepared by carbothermic reduction have excellent morphology and surface characteristics. They are more suitable for hot pressing and hot isostatic pressing.
In the present work, a specially designed graphite resistance furnace has been used to produce boron carbide on a pilot scale. The effects of charge stoichiometry on final powder composition and charge type on powder forming efficiency have been studied. The effect of salt addition as a catalyst in a boron carbide formation reaction has been investigated. The result of arc and resistive heating conditions has been analysed in terms of product compositions and reaction efficiencies.

\section{EXPERIMENTAL}

The experiments were performed on mixtures made from locally available boric acid and petroleum coke, including small amounts of a proprietary catalyst to activate the reaction. The constituents were weighed and mixed in several desired boric acid/petroleum coke ratios such that the charges have 11,15 and $18 \%$ excess amounts of $\mathrm{H}_{3} \mathrm{BO}_{3}$ to accommodate the vapourisation losses of boron and to change the boron content of boron carbide powder. The charges were mixed in 25 $\mathrm{kg}$ batches in a rotary mixer with the addition of a small amount of water to enhance the mixing. The total amount of charge prepared varied from 80 and $145 \mathrm{~kg}$ per experiment, depending on the duration of the experiment. Furthermore, a known amount of recycled mass from the previous experiment was also added to the furnace during the experiment.

A schematic diagram of the furnace used in the present experiments is shown in Fig. 1. The furnace is made of mild steel with a water-cooled copper jacket. The interior is lined with graphite. Current is supplied to the graphite heating element through two insulated terminal blocks to which water-cooled connectors are attached. The heating element is machined from electric arc furnace grade graphite electrode to $4 \mathrm{~cm}$ diameter and $100 \mathrm{~cm}$ length. Before charging the furnace, the heating element is carefully placed between the two terminal blocks. The power supply to the furnace was drawn from a high current $(2000 \mathrm{~A}$, $100 \mathrm{~V})$ single phase transformer. The variation of voltage and current values in the furnace were recorded as a function of time during the experiment.

Boron carbide reaction takes place in three different 


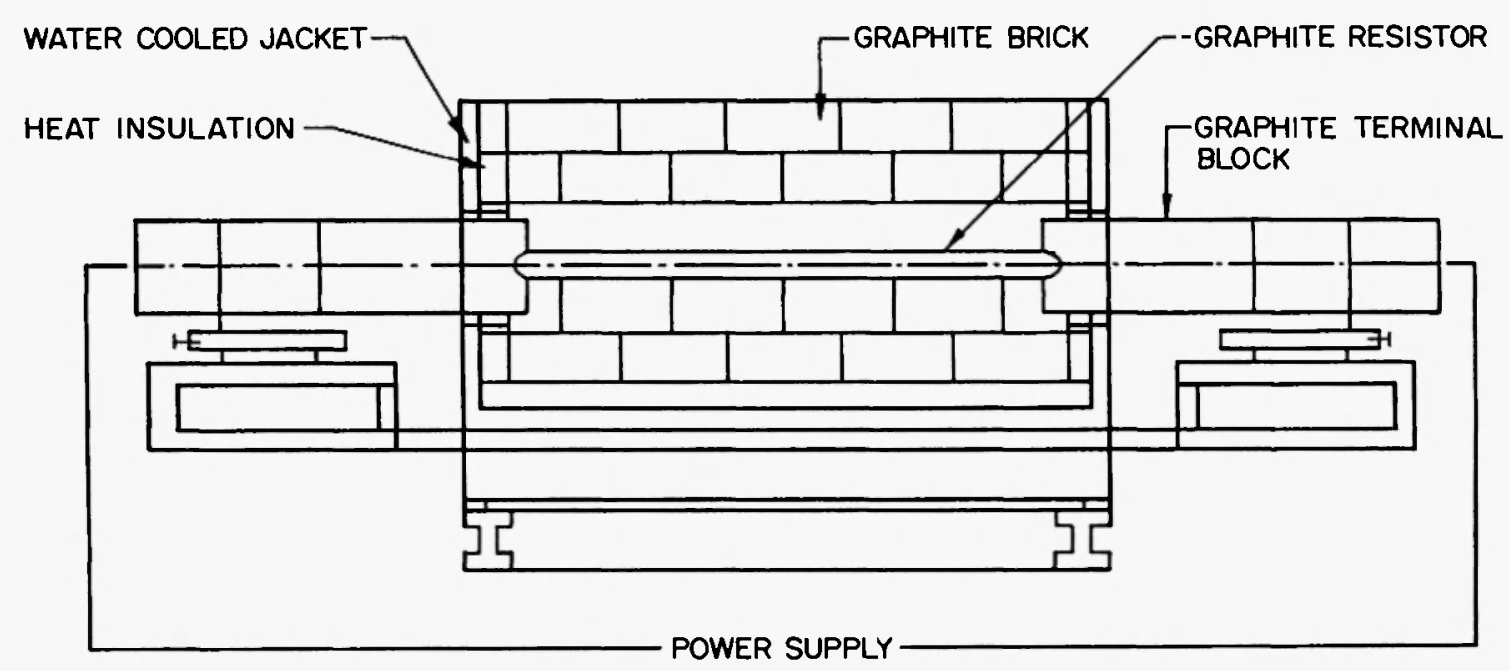

Fig. 1: Schematic diagram of the boron carbide furnace.

stages. In the initial stage of the reaction, the current is kept low to allow the boric acid to dehydrate and form $\mathrm{B}_{2} \mathrm{O}_{3}$. After this the current is increased to initiate the reduction process. The reduction reaction starts with a bluish flame. As the reaction proceeds, the upper surface of the charge was broken at several locations to load extra amounts of new and recycled charge in known quantities. During the experiment, the graphite heating element is consumed slowly and its crosssectional area decreases. This results in an increase in its resistance. The voltage, the current density and the temperature thereby increase. After approximately 5 hours of resistive mode operation, the electrode breaks and the already formed partially fused boron carbide begins to act as a heating element unless an arc is initiated across the broken electrode. Usually the heating is continued until the surface reaches a viscous state and a white yellow flame is no longer visible over the charge. After this stage, the furnace is allowed to cool overnight. The product mass is broken with a pneumatic hammer to recover fused boron carbide chunks and powder. Special care is taken not to mix the partly and fully reacted portions of the product, so that carbon contamination is avoided. The chunk and the powder of the boron carbide are crushed separately in conventional mills, after which dry magnetic separation is applied to remove iron impurity. In the final stage, a tungsten carbide vibrational ring mill is used to make fine boron carbide powder. The powder is cleaned by leaching with a dilute hot acid solution to remove unreacted oxide from the product.

\section{RESULTS AND DISCUSSION}

The analysis of boron carbide products obtained in different experiments is given in Table 1 . The chemical composition of the products indicates the strong influence of charge stoichiometry $\left(\mathrm{H}_{3} \mathrm{BO}_{3}\right.$ to $\mathrm{C}$ ratio) on the product quality. An increase in the $\mathrm{H}_{3} \mathrm{BO}_{3}$ to $\mathrm{C}$ ratio results in an increase in the boron content of the product and also a decrease in its free carbon content. This would be anticipated from the fact that, at higher ratios, more boron is available to result in boron-rich stoichiometries. The maximum boron concentration achieved was $78.6 \%$ and this resulted from a charge with $\mathrm{H}_{3} \mathrm{BO}_{3} / \mathrm{C}$ ratio of 3.6. The product did not contain any free carbon, and all of $20.3 \%$ was combined. Boron carbide of almost this same composition was also obtained even when the chargc contained much less $\mathrm{H}_{3} \mathrm{BO}_{3}$, i.c., even when the $\mathrm{H}_{3} \mathrm{BO}_{3} / \mathrm{C}$ ratio was only 3.45. This carbon content is less than the solubility limit of carbon in $\mathrm{B}_{4} \mathrm{C}$.

With boric acid as the starling material, the boron carbide formation reaction can be represented as

$$
4 \mathrm{H}_{3} \mathrm{BO}_{3}+7 \mathrm{C}=\mathrm{B}_{4} \mathrm{C}+6 \mathrm{CO}+6 \mathrm{H}_{2} \mathrm{O}
$$


Table 1

Chemical analysis in wt\% of boron carbide powder produced using different charge stoichiometries

\begin{tabular}{|c|c|c|c|c|c|c|c|c|}
\hline \hline Experiment No. & $\mathrm{H}_{3} \mathrm{BO}_{3} / \mathrm{C}$ & $\mathrm{B}_{\text {total }}$ & $\mathrm{C}_{\text {total }}$ & $\mathrm{C}_{\text {tree }}$ & $\mathrm{S}$ & $\mathrm{Fe}_{2} \mathrm{O}_{3}$ & $\mathrm{SiO}_{2}$ & $\mathrm{~B}_{2} \mathrm{O}_{3}$ \\
\hline 3 & 3.3 & 43 & 43 & 27.04 & - & - & - & - \\
\hline 5 & 3.3 & 42 & 44 & 28.32 & - & - & - & - \\
\hline 9 & 3.3 & 71.4 & 24.6 & 3.48 & 0.2 & 0.43 & - & 0.64 \\
\hline 10 & 3.3 & 69.8 & 24.1 & 2.84 & 0.43 & 0.6 & 2.36 & 0.64 \\
\hline 11 & 3.6 & 78.6 & 20.3 & - & 0.08 & 0.14 & 0.47 & - \\
\hline 12 & 3.45 & 76.5 & 20.47 & - & 0.03 & - & 0.34 & - \\
\hline 13 & 3.45 & 77.1 & 21.25 & - & 0.04 & 0.09 & 0.32 & - \\
\hline
\end{tabular}

The weight loss associated with the reaction is $83.4 \%$. The reduction efficiency can therefore be calculated by calculating the mass balance in the experiments. The results are given in Table 2. Experiment 1 listed in the table was performed under arc conditions in the later stages. Experiments 2, 3 and 5 were carried out under resistive load conditions to avoid boron losses due to the high arc temperatures. All experiments were performed with boric acid except experiment 5 in which $\mathrm{B}_{2} \mathrm{O}_{3}$ was used as the charge. These results indicate that arc heating conditions result in lower reaction efficiency than resistive heating for the same charge. This is illustrated by experiments 1 and 2 . The charge stoichiometry also has marked influence on the reduction efficiency in certain ranges. The reduction efficiency decreased by about $38 \%$ when the $\mathrm{H}_{3} \mathrm{BO}_{3} / \mathrm{C}$ ratio was increased from 3.3 to 3.45 . Further increase of this ratio to 3.6 , however, did not affect the efficiency much. The deficiency in the reductant availability probably causes such decrease in reduction efficiency. The use of $\mathrm{B}_{2} \mathrm{O}_{3}$ in place of $\mathrm{H}_{3} \mathrm{BO}_{3}$ also causes a marked decrease in reduction efficiency. $\mathrm{A}$ glassy $\mathrm{B}_{2} \mathrm{O}_{3}$ skin formation on the charge surface comes in the way of $\mathrm{CO}$ escape from the reduction zone and thus affects efficiency.

Interesting results were obtained when varying amounts of salt $(\mathrm{NaCl})$ were added to the charge mixture consisting of $\mathrm{H}_{3} \mathrm{BO}_{3}$ and petroleum coke. It was found that salt addition results in a porous structure on a microscale and in a decrease in the specific energy consumption for $\mathrm{B}_{4} \mathrm{C}$ production. The dependence of the specific energy consumption for $\mathrm{B}_{4} \mathrm{C}$ production on the quantity of salt added is depicted in Fig. 2. There is an optimum concentration of the salt at which the energy consumption is minimum. The optimum value of salt concentration is $1.5 \%$. In order to assess the amount of energy required for $\mathrm{B}_{4} \mathrm{C}$ formation, two experiments were carried out with a mixture having 3 and $5.8 \%$ salt content. There was a dramatic increase in energy consumption with the charge using a higher percentage of salt. This probably results from the presence of chloride ions in the salt which may have a corrosive effect on the graphite heater.

Table 2

Experimental efficiencies based on charge and product amounts

\begin{tabular}{|c|c|c|c|c|}
\hline Experiment No. & $\mathrm{H}_{3} \mathrm{BO}_{3} / \mathrm{C}$ & Charge, $\mathrm{kg}$ & Product, $\mathrm{kg}$ & Efficiency, \% \\
\hline 1 & 3.3 & 80 & 10.1 & 75.3 \\
\hline 2 & 3.3 & 130 & 20.0 & 92.7 \\
\hline 3 & 3.45 & 127 & 11.5 & 54.5 \\
\hline 4 & 3.6 & 144.5 & 12.6 & 52.6 \\
\hline 5 & 3.3 & 105 & 7.0 & 40.0 \\
\hline
\end{tabular}




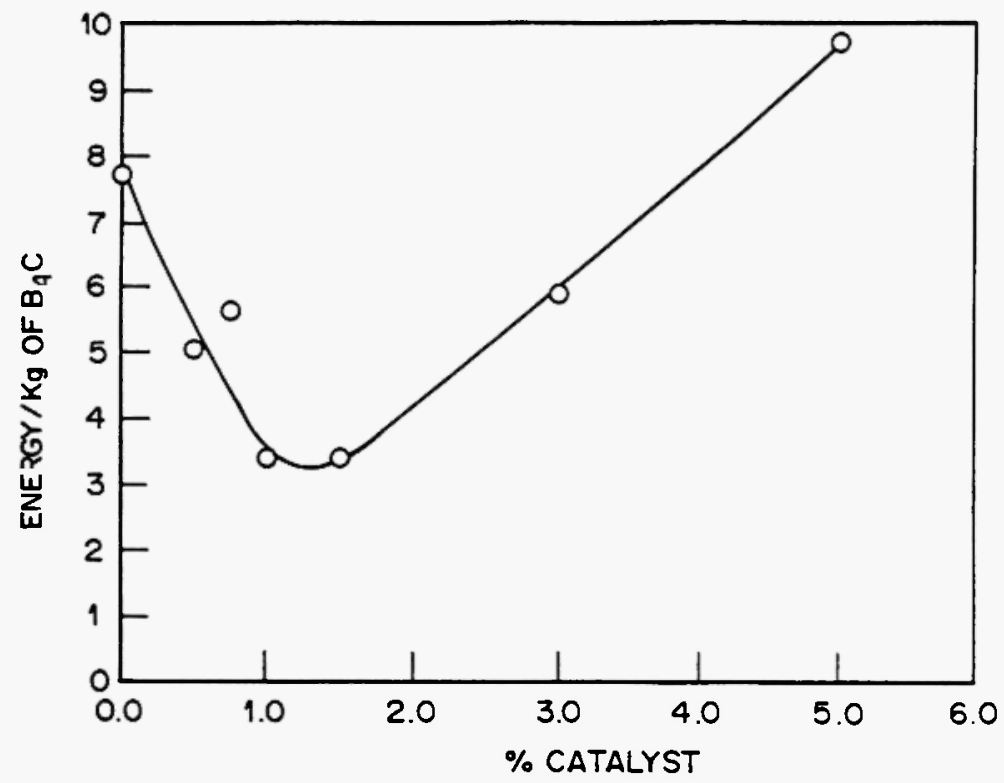

Fig. 2: Specific energy consumption as a function of catalyst percentage in the boric acid petroleum coke charge.

Details of the effect of salt addition and charge composition on the yield of boron carbide are summarised in Table 3. The average energy consumption for all these experiments was about $30 \mathrm{kWh} / \mathrm{kg}$. The reduction efficiency is the best, $92.7 \%$, when the salt addition is $1.5 \%$ and the $\mathrm{H}_{3} \mathrm{BO}_{3} / \mathrm{C}$ ratio is 3.3. The same charge in a similar system converts to boron carbide with only $72 \%$ efficiency consuming- $36 \mathrm{kWh} / \mathrm{kg}$ energy when no salt was added $/ 6 /$.
It was observed in all the experiments that boron carbide forms around the heating element and grows into the liquid phase. The columnar structure perpendicular to the graphite resistor surface indicates that the resistor may be functioning as the seeding material. Whiskers of boron carbide are found in locations which do not have any direct contact with the liquid phase. This probably results from gas-phase reduction. Thus boron carbide formation takes place

Table 3

Experimental efficiencies based on charge and product amounts

\begin{tabular}{|c|c|c|c|c|c|}
\hline No. & Catalyst, \% & $\mathrm{H}_{3} \mathrm{BO}_{3} / \mathrm{C}$ & Charge, $\mathrm{kg}$ & Product, $\mathrm{kg}$ & Efficiency, \% \\
\hline 1 & 5 & 3.3 & 50 & 4.6 & 55 \\
\hline 7 & 0 & 3.3 & 50 & 5.2 & 62.5 \\
\hline 8 & 0.5 & 3.3 & 90 & 9.5 & 63.5 \\
\hline 9 & 0.75 & 3.3 & 80 & 10.0 & 75.3 \\
\hline 10 & 1.5 & 3.3 & 130 & 20.0 & 92.7 \\
\hline 11 & 1.5 & 3.6 & 144 & 12.6 & 52.6 \\
\hline 12 & 1.5 & 3.45 & 148 & 9.7 & 39.5 \\
\hline 13 & 1.5 & 3.45 & 127 & 11.5 & 54.5 \\
\hline $14^{*}$ & 1.5 & 3.3 & 105 & 7.0 & 40.0 \\
\hline
\end{tabular}

Used $\mathrm{B}_{2} \mathrm{O}_{3}$ instead of $\mathrm{H}_{3} \mathrm{BO}_{3}$ 
not only through liquid-phase reduction but also via a gas-phase condensation mechanism.

\section{CONCLUSIONS}

Boron carbide can be produced by carbothermic reduction (using petroleum coke) of boric acid or boron anhydride in an electric resistance furnace where the powder charge mixture is packed around the graphite rod resistor. The charge composition, i.e., the ratio $\mathrm{H}_{3} \mathrm{BO}_{3} / \mathrm{C}$ determines the chemical composition of the product and also the yield of boron carbide, while a product with the highest boron content and no free carbon is obtained with a charge having a $\mathrm{H}_{3} \mathrm{BO}_{3} / \mathrm{C}$ ratio of 3.6; the yield/reduction efficiency was maximum when the ratio was kept at 3.3. Development of arc heating conditions during reduction decreases the yield substantially. There is, however, no effect on the chemical composition of the product. For a different reason, decrease in reduction efficiency occurs when $\mathrm{B}_{3} \mathrm{O}_{3}$ instead of $\mathrm{H}_{3} \mathrm{BO}_{3}$ was used in the charge. The use of an optimum $1.5 \mathrm{wt} \%$ sodium chloride as additive to the charge results in a very marked improvement in reduction efficiency, thus decreasing the specific energy requirement for boron carbide formation by $20 \%$.

\section{REFERENCES}

1. G.A. Meerson and G.V. Samsonov, Proc. Conf. on Boron and Its Compounds, M. GHI, USSR, 1958; p. 52.

2. G.V. Samsonov, Ukr. Chem. J., 24, 799 (1958).

3. R. Swindell and D.E. Lloyd, Hot pressed boron carbide armour, British Ceramic Limited Research Paper, ISBN 0948593 39, 3 February 1989.

4. L. Ya. Markovskii, D.L. Orshanskii and V. Prianishnikev, Electrothermic Chemistry $M-L$, USSR: Goshimizdat, 1952.

5. F. Thevenot, Boron carbide - a comprehensive review, J. European Ceramic Soc., 6, 205 (1990).

6. D.K. Bose, K.U. Nair and C.K. Gupta, High Temperature Materials and Processes, 7, 133 (1986). 